ARTICLE

\title{
Forward and reverse genetic dissection of morphogenesis identifies filament-competent Candida auris strains
}

\author{
Darian J. Santana (iD ${ }^{1} \&$ Teresa R. O'Meara (iD ${ }^{1 凶}$
}

Candida auris is an emerging healthcare-associated pathogen of global concern. Recent reports have identified $C$. auris isolates that grow in cellular aggregates or filaments, often without a clear genetic explanation. To investigate the regulation of $C$. auris morphogenesis, we applied an Agrobacterium-mediated transformation system to all four C. auris clades. We identified aggregating mutants associated with disruption of chitin regulation, while disruption of ELM1 produced a polarized, filamentous growth morphology. We developed a transiently expressed Cas 9 and sgRNA system for $C$. auris that significantly increased targeted transformation efficiency across the four $C$. auris clades. Using this system, we confirmed the roles of $C$. auris morphogenesis regulators. Morphogenic mutants showed dysregulated chitinase expression, attenuated virulence, and altered antifungal susceptibility. Our findings provide insights into the genetic regulation of aggregating and filamentous morphogenesis in C. auris. Furthermore, the genetic tools described here will allow for efficient manipulation of the $C$. auris genome.

\footnotetext{
${ }^{1}$ Department of Microbiology and Immunology, University of Michigan Medical School, Ann Arbor, MI, USA. 凶email: tromeara@umich.edu
} 
S ince its 2009 isolation from the ear canal of a patient in Japan, the emerging fungal pathogen Candida auris has caused infections and outbreaks in at least 44 countries on 6 continents $^{1}$. The global prevalence of $C$. auris is characterized by the seemingly simultaneous emergence of four distinct genetic clades, differing on the scale of hundreds of thousands of single nucleotide polymorphisms (SNPs), with a potential fifth clade recently identified ${ }^{2,3}$. Individual isolates exhibit significant heterogeneity both within and between clades, including in murine models of infection and colonization ${ }^{4,5}$. The continually increasing understanding of biologically and clinically relevant phenotypic variation among $C$. auris isolates, and the variation between $C$. auris and other well-studied model organisms, emphasizes the need for facile genetic manipulation approaches to allow for mechanistic characterization of this organism.

Although $C$. auris does not form filaments under many of the same environmental cues that induce hyphal growth in Candida albicans $^{6}$, numerous reports of irregular or multicellular growth indicate C. auris does exhibit cellular polymorphism. Depletion of the essential molecular chaperone HSP90 results in elongated cell growth ${ }^{6}$. Genotoxic stress induced by hydroxyurea or deletion of the DNA damage responsive long non-coding RNA DINOR similarly result in pseudohyphal elongated cells ${ }^{7,8}$. Other stressors such as growth in high salt concentrations induce cell elongation ${ }^{9}$. Strains exhibiting filamentous, elongated, or aggregating morphologies have been isolated from populations of C. auris cells following murine infection ${ }^{10,11}$. Furthermore, numerous reports detail patient isolates with multicellular aggregating phenotypes, often described by a failure of cell aggregates to disperse upon mixing or vortexing ${ }^{12-15}$. Aggregating isolates exhibit reduced biomass in biofilm formation and lower virulence in Galleria mellonella infection models compared to non-aggregating counterparts ${ }^{12,16}$. Still, the genetic determinants of irregular morphogenesis in C. auris remain largely unexplored due in part to difficulties in performing genetic manipulation in this organism.

Transformation of $C$. auris is complicated by low rates of targeted integration and variable transformation efficiency among isolates and clades ${ }^{7,17}$. The use of RNA-protein complexes of purified Cas9 and gene-specific guide RNAs, referred to as Cas9ribonucleoproteins (RNPs), to promote homology directed repair demonstrably increases transformation efficiency and targeted integration rates ${ }^{18}$. Transformation incorporating RNPs is often the method of choice for manipulating the C. auris genome, and variations exist using multiple gRNA target sites to further improve targeted integration efficiency ${ }^{19}$. The use of RNPs in transformation, however, comes with increased expense and additional technical considerations during transformation. In $C$. albicans, transformation with linearized gene cassettes encoding Cas9 and sgRNA promote homozygous gene deletion; these cassettes cannot be detected in the genome of transformants, suggesting they are transiently expressed and not stably integrated $^{20}$. A similar transiently expressed CRISPR-Cas9 system promotes targeted genetic manipulation in Cryptococcus neoformans ${ }^{21}$. We hypothesized that specific adaptation of the transiently expressed CRISPR-Cas9 system to use C. aurisrecognized promoters would increase the rates of targeted transformation efficiency.

A forward genetics system represents an alternative approach for manipulating the genome. The piggyBac transposon mutagenesis system has proven successful for performing insertional mutagenesis at saturating levels in a Clade II C. auris isolate ${ }^{8}$. This represents a significant advance in the technical ability to genetically manipulate $C$. auris. However, one potential limitation of the piggyBac system is that it requires initial engineering of the strain of interest to encode the transposon machinery prior to performing genome-scale mutagenesis. To develop a forward genetics system suitable for performing mutagenesis in any C. auris clinical isolate without prior engineering, we turned to Agrobacterium tumefaciens-mediated transformation (AtMT), an insertional mutagenesis approach with a history of proven success in fungal species ${ }^{22}$. A. tumefaciens is a plant pathogen that causes crown gall in dicotyledonous plants through genetic transformation ${ }^{23}$. Its capacity for transformation is not limited to plants, however, and can be taken advantage of to perform insertional transformation in a variety of eukaryotic species, including C. albicans, Candida glabrata, and Saccharomyces cerevisiae $^{24}$. In practice, mobilization of a DNA sequence flanked by left and right direct repeats (T-DNA) is accomplished by induction of $A$. tumefaciens virulence genes during co-culture with a recipient organism using acetosyringone ${ }^{25}$. This T-DNA sequence is encoded on the Ti Plasmid harbored by A. tumefaciens and can be manipulated to contain fungal selectable markers.

We used AtMT to generate an insertional mutant library in C. auris and identified morphogenic mutants exhibiting aggregating or filamentous growth. Insertions in genes orthologous to regulators of chitinase and chitin synthase in $S$. cerevisiae were associated with defects in daughter cell separation in C. auris, leading to aggregating growth, while an insertion in an ortholog of ScELM1 resulted in constitutive filamentous growth in C. auris. We developed a robust transient CRISPR-Cas9 expression system for $C$. auris and demonstrated its ability to significantly increase targeted transformation in isolates from all four major clades. Using this system, we performed deletions in key regulators of cell separation to demonstrate functional conservation of ELM1 and chitin regulatory genes as morphogenic regulators in C. auris. The morphogenic mutants we identified exhibited attenuated virulence in a G. mellonella infection model and altered antifungal susceptibility profiles. The tools presented here allowed for detailed analyses of the genetic circuitry required for morphogenesis in the emerging pathogen $C$. auris and will serve as a resource to the community for future molecular genetic manipulation of this pathogen.

\section{Results}

Agrobacterium-mediated transformation identifies C. auris morphogenic mutants. While aggregating and filamentous strains of $C$. auris have been recovered from human and murine hosts, the genetic circuitry governing $C$. auris morphogenesis remains largely uncharacterized. Therefore, we set out to apply a forward genetic approach to identify regulators of morphogenesis in C. auris. To accomplish this, we developed an AtMT system for C. auris. We cloned the CaNAT1 nourseothricin resistance cassette into the pPZP Ti plasmid backbone between the T-DNA left and right borders to generate pTO128 (pPZP-NATca) and transformed the resulting vector into $A$. tumefaciens strain EHA105, which also harbors the virulence genes necessary for mobilization of the T-DNA. We used representative $C$. auris clinical isolates from the FDA-CDC Antimicrobial Resistance Isolate $\mathrm{Bank}^{26}$ to measure the transformation efficiency of AtMT in each of the four major C. auris clades (Fig. 1a). By comparing the number of recovered nourseothricin-resistant transformants to the number of input cells, we observed successful transformation in isolates from all four clades with variable transformation efficiency. Of the isolates tested, we observed the highest transformation efficiency in the Clade I isolate AR0382, with an average efficiency of $0.16 \%$ ( 1 in 625 C. auris cells) (Fig. 1a). The Clade II isolate AR0381 showed the lowest transformation efficiency at $0.0025 \%$ ( 1 in 40,000 C. auris cells) under the same growth conditions, though even this rate is consistent with the 
a

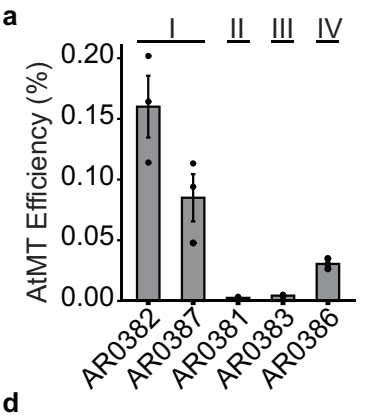

b

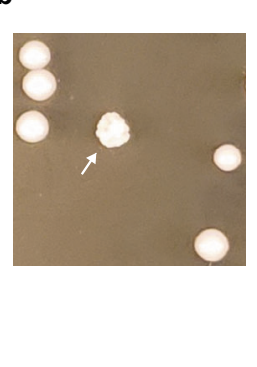

C

\begin{tabular}{|c|c|c|}
\hline Strain & Clade & $\begin{array}{c}\text { Filamentous } \\
\text { Transformants (\%) }\end{array}$ \\
\hline AR0382 & $\mathrm{I}$ & $9 / 5615(0.0016)$ \\
\hline AR0387 & I & $9 / 2910(0.003)$ \\
\hline AR0381 & II & 4/1346 (0.003) \\
\hline AR0383 & III & $5 / 4768(0.001)$ \\
\hline AR0386 & IV & $14 / 2021(0.007)$ \\
\hline
\end{tabular}

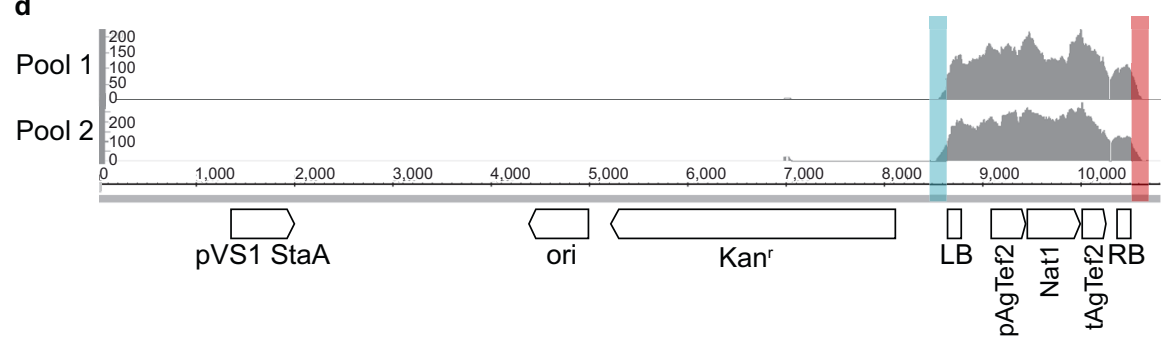

Fig. 1 Agrobacterium tumefaciens-mediated transformation (AtMT) identifies regulators of colony morphology in C. auris. a AtMT transformation efficiency of $C$. auris was measured for representative isolates from each of four major clades. Transformation efficiency is expressed as the ratio of recovered $C$. auris transformants to the total number of input $C$. auris cells. Data shown are mean \pm standard error of the mean from three independent experiments. Source data are provided as a Source Data file. b Morphogenic mutants were identified in C. auris AtMT transformants through irregular colony morphologies (arrow). c The rates of morphogenic mutants recovered for insertional mutants from representative isolates from each of four major clades are expressed as the ratio of morphogenic mutant CFU to total CFU screened for aberrant morphology. $\mathbf{d}$ Genomic DNA was extracted from 6 morphogenic mutants from AR0382 (Clade I) and pooled into two pools of 3 for Illumina sequencing. Reads were mapped to the TI Plasmid ( $p$ TO128), represented as a read coverage plot. Highlighted regions in blue and red indicate read sequence that extended beyond the T-DNA left and right borders, respectively, used to identify transgene insertion sites in the $C$. auris genome.

range of transformation efficiencies exhibited in integrative AtMT of other yeast species (Fig. 1a) ${ }^{27,28}$. We visually screened recovered transformants for those with altered colony morphology, suggestive of an alteration in cellular morphology (Fig. 1b). In this manner, we identified morphological mutants in isolates from all four major clades. The rate at which we recovered morphological mutants differed significantly among the clades (Chi sq. $=22.42$, $\left.p=1.66 \times 10^{-4}\right)$, with the highest rate in the Clade IV isolate AR0386 (Fig. 1c). These findings demonstrate the utility of AtMT as a forward genetics system to discover mutant phenotypes in all four major clades of $C$. auris.

Transgene insertion sites can be defined by identifying the genomic regions flanking the insertions using whole-genome sequencing 29 . We reasoned a similar approach could identify transgene insertion sites from multiple mutants sequenced in pools. We mapped Illumina sequencing reads from two pools of three morphogenic mutants selected from AtMT of AR0382 (Clade I) to the sequence of the TI plasmid pTO128 (pPZPNATca). The sequencing reads mapped exclusively to the T-DNA region of the plasmid, demonstrating the specificity of the integration, with additional read length spanning either junction at the T-DNA left and right borders (Fig. 1d). The sequence extending beyond the left and right borders corresponded to $C$. auris genomic regions flanking the transgene insertions. We deconvoluted the pools using standard PCR and Sanger sequencing with insertion site-specific primers.

Among the mutants identified by irregular colony morphologies, four exhibited a similar aggregating cellular phenotype, with individual cells connected into clusters that could not be disrupted by vortexing (Fig. 2). Insertion events in CauACE2 (B9J08_000468), orthologous to S. cerevisiae ACE2 (YLR131C), as well as in CauTAO3 (B9J08_000181), orthologous to S. cerevisiae TAO3 (YIL129C), were associated with this aggregatory phenotype. A similar aggregating phenotype resulted from an insertion near the C-terminus of CauCHS2 (B9J08_003879), an ortholog of
CHS2 (YBR038W) in S. cerevisiae. A fourth aggregating strain was associated with an insertion in the upstream region of B9J08_002252; however, orthologs of this gene in related species are poorly characterized. To predict a potential function for this gene, we analyzed the C. albicans ortholog C7_00260C using the CalCEN Co-expression network ${ }^{30}$. GO term analysis revealed that 43 of 50 co-expressed genes fall under the "piecemeal microautophagy of the nucleus" term (Supplementary Fig. 1). We also observed pseudohyphae-like filaments characterized by elongated cells with constricted separations between compartments in a mutant with an insertion in CauELM1 (B9J08_002849), an ortholog of S. cerevisiae ELM1 (YKL048C) (Fig. 2).

A sixth insertional mutant identified by its irregular colony morphology exhibited similar aggregating growth (Supplementary Fig. 2). For this mutant, we identified T-DNA sequence both in the intergenic space upstream of the B9J08_002954 ORF and in the intergenic region upstream of the B9J08_002667 ORF from the B8441 reference sequence, but we were unable to amplify the complete insertion locus of either site from genomic DNA of the mutant. We hypothesize that a recombination event or other chromosomal rearrangement may have occurred following one or multiple T-DNA insertion events in this mutant, though further investigation is required to confirm this. Together, these findings identify key components of the regulation of cell separation in $C$. auris.

Expression of Cas9 and sgRNA increases targeted integration in C. auris. To validate the insertional mutagenesis and confirm the role of identified genes in regulating the multicellular phenotypes we observed, we sought to recapitulate the phenotypes via clean deletions of the target genes. However, targeted homologous recombination has low efficiency in C. auris, adding considerable technical challenge to performing 


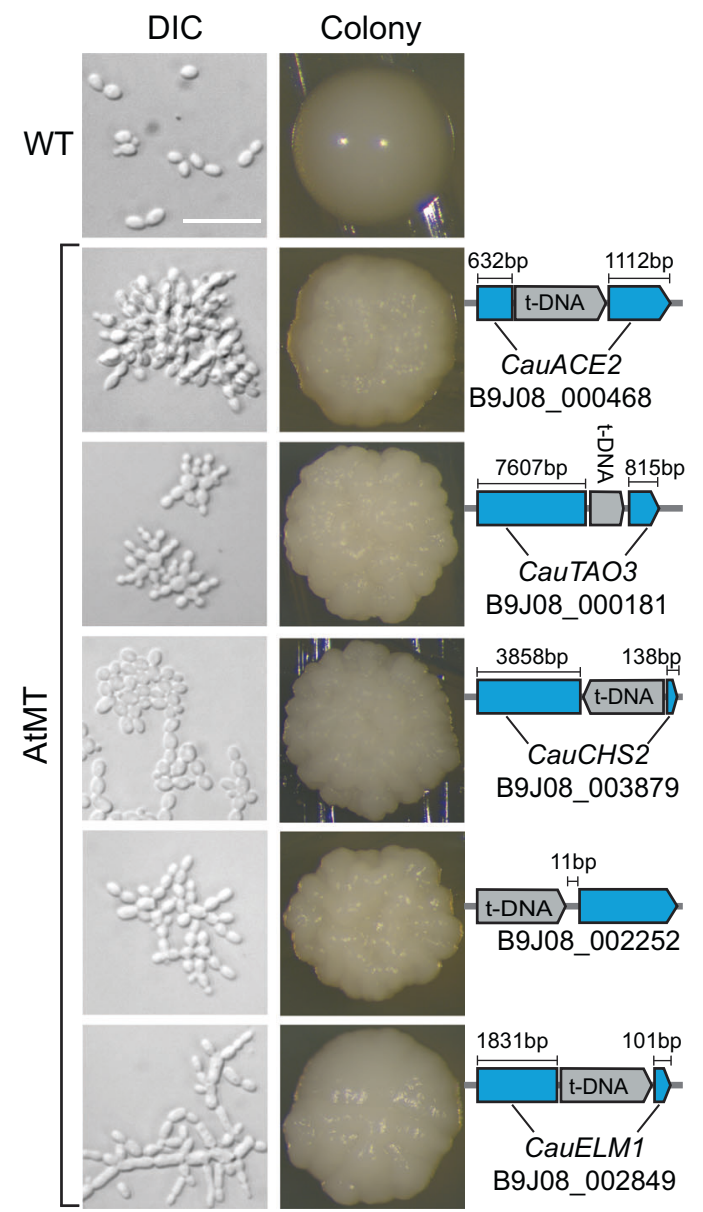

Fig. 2 Transgene insertion sites associated with C. auris morphogenic mutants. Cell (DIC microscopy, Differential Interference Contrast) and colony morphologies demonstrate distinct morphogenic defects in five AtMT (Agrobacterium tumefaciens-mediated transformation) insertional mutants (bottom) compared to wild-type C. auris AR0382 (top). Identified transgene insertion sites were confirmed using Sanger sequencing (right). In all five cases, T-DNA insertion events were not accompanied by any additional insertions or deletions in the insertion locus. Scale bar $=20 \mu \mathrm{m}$.

genetic manipulation ${ }^{7,17}$. Transformation in C. auris can be facilitated by the use of Cas 9 and sgRNA ribonucleoproteins; however, a previous DNA-based transient CRISPR-Cas9 expression approach used in C. albicans does not substantially improve targeted transformation efficacy in C. auris (ref. ${ }^{6}$ and personal communication, Sang $\mathrm{Hu}$ Kim). Recently, $\mathrm{Ng}$ and Dean $^{31}$ reported variable increases in targeted transformation efficiency in C. albicans when using different promoters to drive the transcription of the sgRNA in a similar system. We hypothesized that the low efficiency of the transient CRISPR system in C. auris may be due to poor recognition of the SNR52 promoter from C. albicans. Therefore, we sought to develop a transient Cas9 and sgRNA expression system that can be used for efficient transformation in C. auris 20,31 . First, we generated expression cassettes for Cas9 and sgRNA using C. auris-specific promoters (Fig. 3a). We placed the CaCas9 cassette, which has been codon-optimized for expression in CTG clade fungi, under control of the C. auris ENO1 promoter and the sgRNA cassette under control of the C. auris $A D H 1$ promoter. However, because this RNA Polymerase II promoter would generate a transcript with a $5^{\prime}$ cap and $3^{\prime}$ polyA tail, ultimately detrimental to the gRNA targeting effciency, we included the $C$. auris $t R N A-A L A$ sequence immediately upstream of the sgRNA and the hepatitis delta virus (HDV) ribozyme sequence immediately downstream of the sgRNA. With this design, we anticipated cleavage at the $3^{\prime}$ end of the tRNA sequence by endogenous RNase A and self-catalyzed cleavage at the $5^{\prime}$ end of the HDV ribozyme ${ }^{32,33}$.

To assess the functional capacity of the Cas9 and sgRNA expression system to increase the efficiency of targeted integration in C. auris, we designed a reporter cassette that would allow for rapid and specific identification of targeted integration events (Fig. 3b). The reporter cassette contained approximately $500 \mathrm{bp}$ of homology to the C-terminus of C. auris ENO1 and genomic sequence immediately downstream of ENO1. We removed the stop codon from the ENO1 C-terminus homologous sequence and fused RFP to the ENO1 C-terminus with a glycine linker. Because the RFP gene had no promoter element, we anticipated transformants would only demonstrate robust fluorescence if the reporter cassette integrated precisely in frame to tag the Eno1 protein and be driven by the endogenous ENO1 promoter. The reporter cassette also included an independently driven nourseothricin resistance (NAT) cassette to allow identification of the total transformant population by selection on nourseothricin, regardless of integration site. To confirm that the reporter cassette specifically identified targeted integration events, we designed a PCR primer set spanning the ENO1-RFP junction and a primer set spanning a region of the ENO1 locus native to the wild type. We performed transformation with the reporter cassette and recovered a representative sample of nourseothricin-resistant transformants that were either fluorescent or non-fluorescent. Amplification of the region spanning the ENO1-RFP junction was only exhibited by the fluorescent transformants and not by the wild-type or non-fluorescent transformants, while amplification of the wild-type sequence was exhibited by all the transformants and the wild-type strain (Fig. 3c). This demonstrates that the ratio of fluorescent to non-fluorescent colonies is a reliable measure of the efficiency of targeted integration.

We observed variable targeted transformation efficiency among C. auris isolates of different genetic backgrounds (Fig. 3d). We therefore sought to determine whether the Cas 9 and sgRNA expression system promoted targeted transformation in multiple genetically diverse $C$. auris isolates (Fig. 3d). The targeted integration rate under each transformation condition was determined by dividing the number of fluorescent colonies by the total number of nourseothricin-resistant transformant colonies. For AR0387, a Clade I isolate, inclusion of the Cas9 and sgRNA expression cassettes increased the targeted integration rate to $44.7 \%$ of transformants from an average of $16.9 \%$ using only the reporter cassette. We observed similar trends for isolates from each clade. Targeted integration increased from 18.2 to $77.8 \%$ in AR0381 (Clade II), from 4.6 to $32.8 \%$ in AR0383 (Clade III), and from 1.8 to $25.5 \%$ in AR0386 (Clade IV) with the addition of the Cas9 and sgRNA expression cassettes compared to the reporter cassette alone (Fig. 3d). The ENO1 C-terminus homologous arm encoded by the reporter cassette showed $100 \%$ sequence identity in all four isolates, while AR0381 and AR0383 shared four nucleotide variants out of $557 \mathrm{bp}$ in the downstream homologous arm and AR0386 showed a single nucleotide variant in the same region (Supplementary Fig. 3). Therefore, differences in the targeted integration efficiency could not be explained by differential homology to the reporter cassette. Moreover, we were unable to detect integration of the CAS9 cassette in a majority of recovered fluorescent transformants across all four clades (Supplementary Fig. 4), suggesting the Cas9 system is transient as designed, with rare integration events, consistent with previous observations from a similar system in $C$. albicans $^{20}$. Taken together, these observations indicate the Cas9 and sgRNA expression cassettes successfully promote targeted transformation in all four major $C$. auris clades. 
a

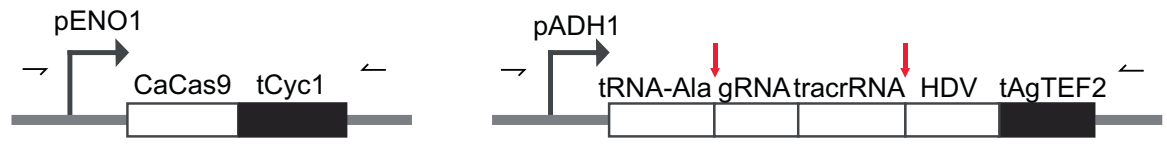

b

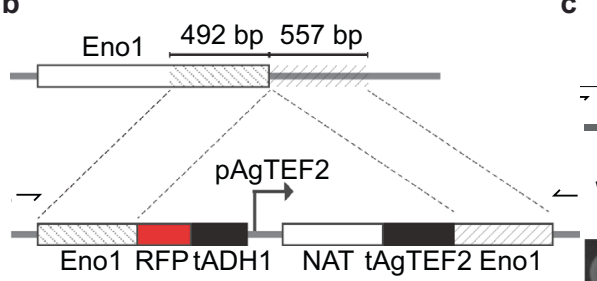

$A$

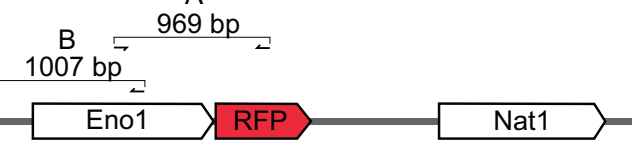

WT Fluorescent

Non-Fluorescent Transformants Transformants

$1 \mathrm{~kb}-\quad \square=00$

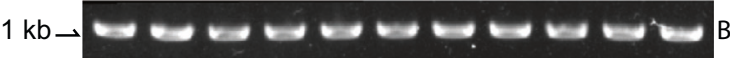

d

AR0387

Clade I

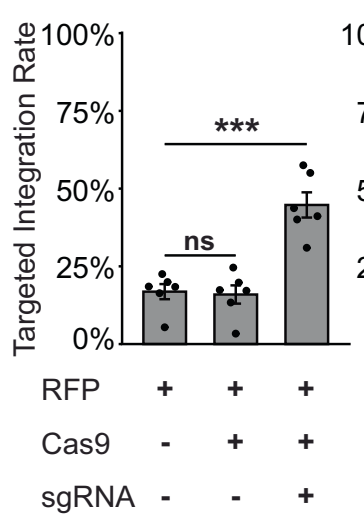

AR0381

Clade II
AR0383

Clade III
AR0386

Clade IV
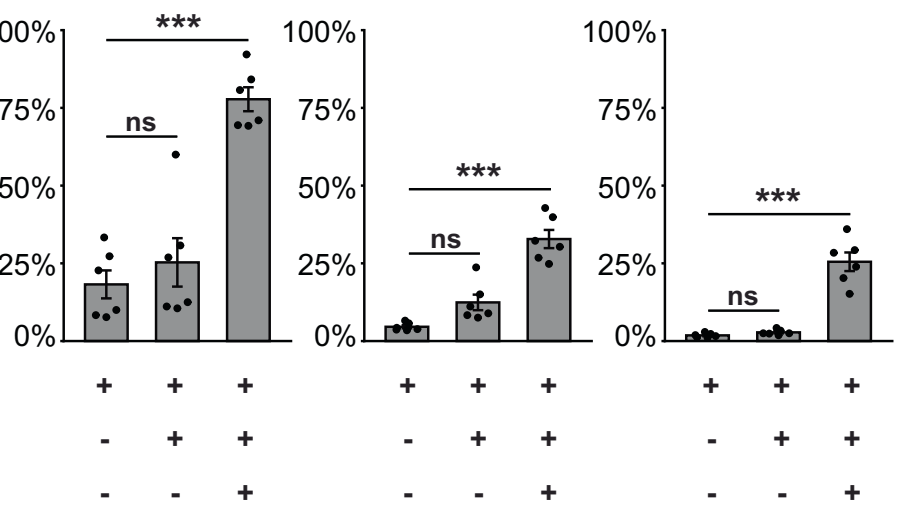

Fig. 3 A CRISPR-Cas9 expression system promotes targeted transformation in four $\mathbf{C}$. auris clades. a Structures of the Cas9 and sgRNA expression cassettes. CAS9 is driven by the $C$. auris ENO1 promoter and followed by the CYC1 terminator. The sgRNA cassette is driven by the C. auris ADH1 promoter and contains C. auris tRNA-Ala immediately upstream of the 20-bp gRNA sequence and hepatitis delta virus (HDV) ribozyme immediately downstream of the tracrRNA sequence. Predicted post-transcriptional cleavage sites are indicated by red vertical arrows. Primer sites to generate linear transformation cassettes are indicated by horizontal arrows. b Design of the reporter cassette for measuring targeted integration. The cassette is flanked by approximately 500-bp homology to the C. auris ENO1 C-terminus minus the stop codon and the region immediately downstream of C. auris ENO1. RFP and the C. auris $A D H 1$ terminator tag the ENO1 gene at the C-terminus via a glycine linker to generate ENO1-RFP in targeted transformants. An independently driven nourseothricin resistance cassette (NAT) allows identification of total transformants, regardless of integration site, by selection with nourseothricin. c Targeted integration events are identifiable by colony fluorescence. Transformation of AR0387 was performed using the reporter cassette described in panel (b). Representative fluorescent transformants and non-fluorescent transformants were spotted onto YPD. Primer set A, spanning the ENO1-RFP junction, shows amplification only from fluorescent transformants. Primer set B, spanning a neighboring wild-type locus, shows amplification from all transformants and the wild type. $\mathbf{d}$ Expression of Cas 9 and sgRNA promotes targeted integration rate. Transformation was performed in representative isolates from all four major C. auris clades with the linear transformation cassettes described in panels (a) and (b). Transformations were performed with and without Cas9 and sgRNA elements; when absent, the cassettes were replaced with an equivalent volume of buffer. Targeted integration rate is expressed as the ratio of fluorescent colonies recovered to total nourseothricin-resistant colonies recovered. Each point represents an individual transformation. Shown are the mean \pm standard error of the mean from three individual experiments, each performed in duplicate. Source data are provided as a Source Data file. Statistical differences were determined using one-way ANOVA with Tukey's post hoc test for multiple comparisons: AR0387: ${ }^{\star \star \star} p=5.4 \times 10^{-5}$, ns: $p=0.98$; AR0381: ${ }^{\star \star \star} p=5.8 \times 10^{-6}$, ns: $p=0.66 ;$ AR0383: ${ }^{\star \star \star} p=6.0 \times 10^{-7}$, ns: $p=0.063 ;$ AR0386: ${ }^{\star \star \star} p=3.0 \times 10^{-7}$, ns: $p=0.92$.

$A C E 2$ and TAO3 are regulators of $C$. auris morphogenesis. Using these tools, we were able to investigate the function of the genes implicated in $C$. auris morphogenic regulation by AtMT. Deletion of ACE2 or TAO3 in AR0382 (Clade I) resulted in constitutively aggregating cells with individual cells connected at septa, suggestive of a failure of budding daughter cells to separate from mother cells (Fig. 4a). We then codon-optimized a G418 resistance gene for CTG-clade expression and found its expression allowed for selection of C. auris on media containing $1 \mathrm{mg} /$ $\mathrm{mL}$ G418. Using this new dominant selectable marker, we were able to complement $\Delta a c e 2$ and $\Delta$ tao 3 mutants with reconstituted versions of the deleted genes replaced in the endogenous loci. The 
a

AR0382

Clade I

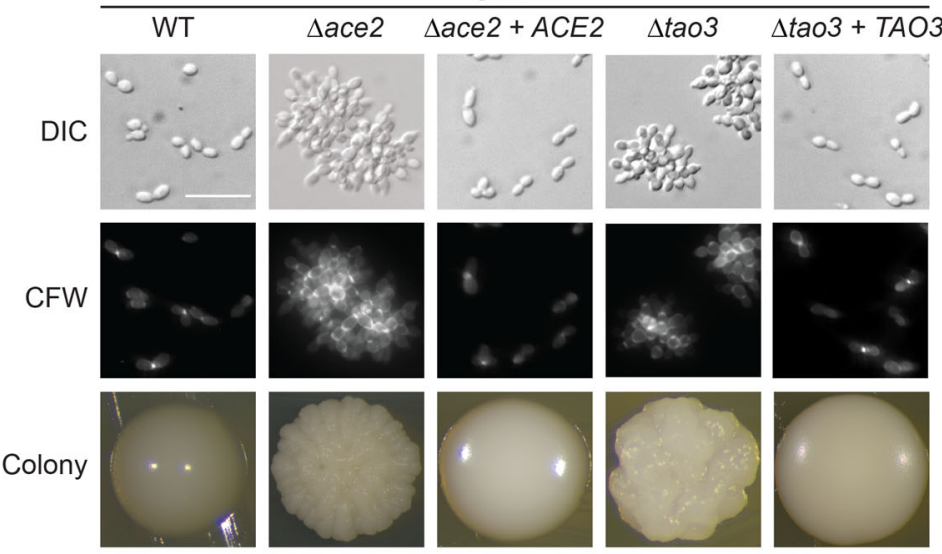

b
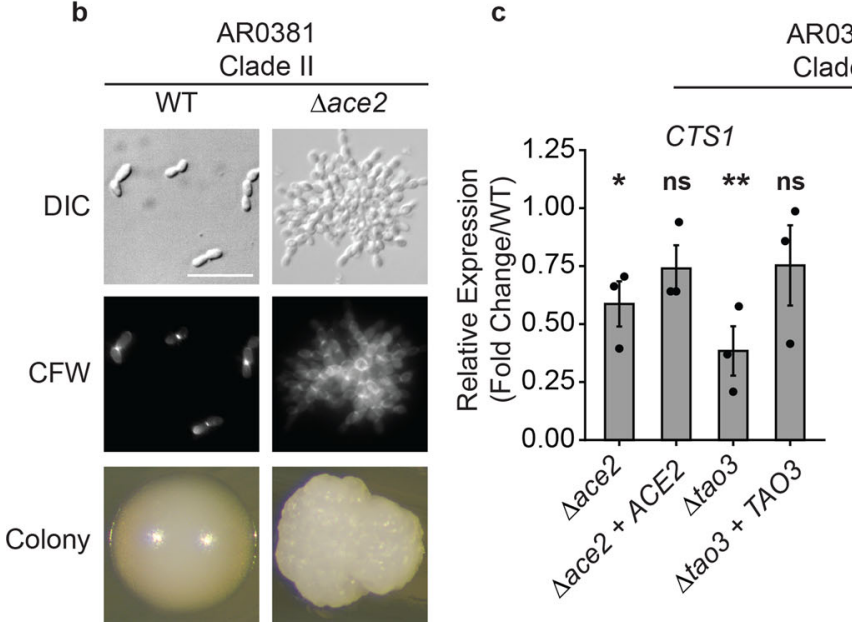

AR0382

Clade I

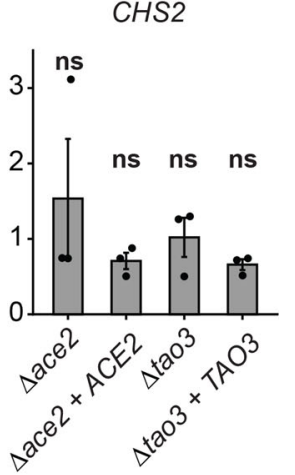

Fig. 4 ACE2 and TAO3 are regulators of $\boldsymbol{C}$. auris morphogenesis. a Microscopy of $\triangle a c e 2, \Delta t a 03$, and complemented strains in the AR382 (Clade I) genetic background. Representative images shown for DIC (Differential Interference Contrast), cells stained with calcofluor white (CFW), and colonies formed on YPD agar. Scale bar $=20 \mu \mathrm{m}$. b ACE2 regulates morphogenesis across C. auris clades. Microscopy of $\triangle$ ace2 and in the AR381 (Clade II) genetic background. Representative images shown for DIC, cells stained with calcofluor white, and colonies formed on YPD agar. Scale bar $=20 \mu \mathrm{m}$. c ACE2 and TAO3 regulate putative chitinase CTS1 but not CHS2 transcription. Wild-type (AR0382), $\Delta a c e 2, \Delta t a 03$, and complemented strains were grown to exponential phase in YPD at $30^{\circ} \mathrm{C}$ prior to RNA extraction and RT-qPCR analysis of upregulated and downregulated genes. Shown are the relative expression of CTS1 and CHS2 for each mutant strain compared to the wild type and normalized to ACT1 gene expression. Mean \pm standard error of the mean from three biological replicates, each with three technical replicates. Strains that showed significantly different expression compared to the wild type are indicated. Source data are provided as a Source Data file. Statistical differences were determined using one-way ANOVA with Dunnett's post hoc test for multiple comparisons. CTS7: $\Delta$ ace2, ${ }^{*} p^{*}+=0.046 ; \Delta a c e 2+A C E 2$, ns: $p=0.25 ; \Delta$ tao3, ${ }^{\star *} p=0.004 ; \Delta$ tao3 + TAO3, ns: $p=0.32$. CHS2: $\Delta a c e 2$, ns: $p=0.87 ; \Delta a c e 2+A C E 2$, ns: $p=0.60$; $\Delta$ tao3, ns: $p=0.93 ; \Delta$ tao3 + TAO3, ns: $p=0.10$

complemented strains $\triangle a c e 2+A C E 2$ and $\triangle$ tao $3+T A O 3$ restored the wild-type cellular and colony morphologies (Fig. 4a). In $S$. cerevisiae, Tao3 associates with kinases Kic1 and Cbk1 as part of the Regulation of ACE2 Morphogenesis (RAM) pathway. Phosphorylation of Ace 2 by Cbk1 results in its accumulation in daughter cell nuclei, where it regulates the expression of enzymes that mediate septum degradation ${ }^{34}$. Mutations in ACE2 or upstream components of the RAM pathway in S. cerevisiae or in C. albicans result in a aggregating, multicellular phenotypes similar to those exhibited by C. auris $\Delta a c e 2$ and $\Delta$ tao 3 mutants, suggesting that $C$. auris has maintained conservation of the RAM pathway in regulating morphogenesis ${ }^{35-37}$. An $\Delta$ ace 2 mutant in AR0381 (Clade II) showed a similar aggregating phenotype, suggesting this role is conserved across $C$. auris clades as well (Fig. 4b). To assess whether the regulation of cell wall maintenance genes was also conserved in $C$. auris, we investigated the transcriptional change in the chitinase gene CTS1 (B9J08_002761), which is homologous to a key enzyme regulated by Ace 2 and responsible for the degradation of the primary septum during daughter cell separation in $S$. cerevisiae ${ }^{38}$. We observed significant downregulation of CTS1 expression in $\triangle a c e 2$ and $\triangle$ tao3 mutants compared to wild-type AR0382, while $\triangle a c e 2+A C E 2$ and $\triangle t a o 3+T A O 3$ mutants showed no significant change in CTS1 expression (Fig. 4c). Because our forward genetics screen suggested disruption of the chitin synthase gene CHS2 could also confer an aggregating phenotype, we asked whether CHS2 expression was altered by deletion of ACE2 or TAO3. However, we observed no significant difference in the expression of CHS2 in $\triangle a c e 2$ or $\Delta$ tao 3 mutants compared to the wild type (Fig. 4c). Together, these findings demonstrate that ACE2 and TAO3 are key regulators of $C$. auris morphogenesis and deletion of either leads to an aggregating phenotype associated with decreased expression of the chitinase gene CTS1.

ELM1 is a regulator of $\boldsymbol{C}$. auris filamentous growth. Next, we investigated ELM1, disruption of which resulted in both an aggregating and elongated cellular phenotype in our AtMT 
a

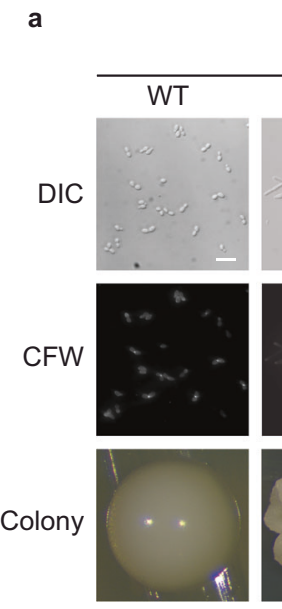

c

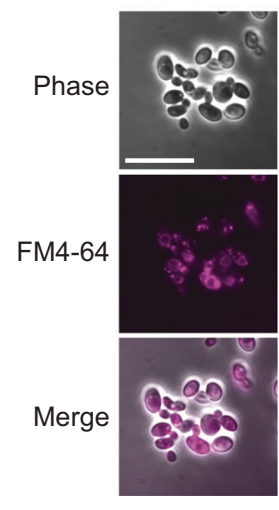

AR0382

Clade I

$\Delta \mathrm{e} / \mathrm{m}$
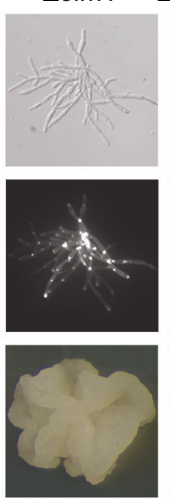

.
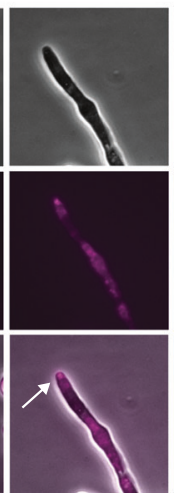
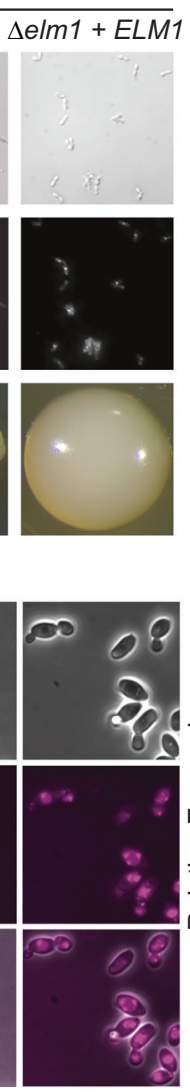

b
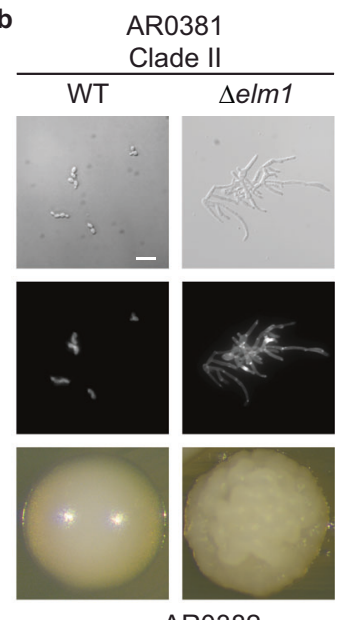

d

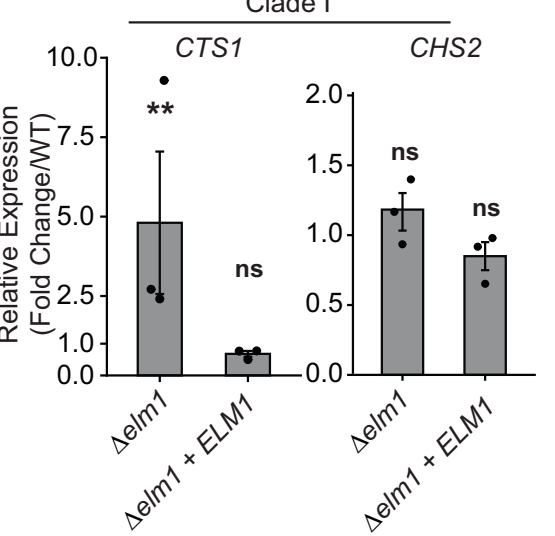

Fig. 5 ELM1 is a regulator of $\boldsymbol{C}$. auris filamentous growth. a Microscopy of wild-type AR0382, $\Delta$ elm1, and complemented $\Delta$ elm $1+E L M 1$ strains. DIC (Differential Interference Contrast), cells stained with calcofluor white (CFW), and colonies formed on YPD agar are shown. Scale bar =10 $\mu$ m. $\mathbf{b}$ ELM1 regulates filamentous growth across $C$. auris clades. Microscopy of DIC, cells stained with calcofluor white, and colonies formed on YPD agar for wild-type

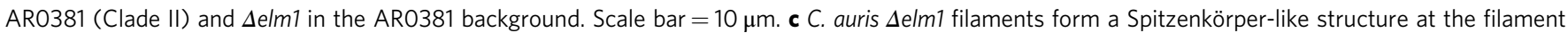
apex. AR0382 (Clade I) wild type, $\Delta$ elm1, and $\Delta$ elm1 + ELM1 strains shown with phase contrast microscopy, stained with the lipophilic dye FM 4-64. Arrow indicates the location of a putative Spitzenkörper where dye has accumulated at the growing filament tip. Scale bar $=10 \mu \mathrm{m}$. $\mathbf{d} E L M 1$ negatively regulates CTS1 but not CHS2 expression. Wild-type (AR0382), $\Delta$ elm1, and $\Delta$ elm1 + ELM1 were grown to exponential phase in YPD at $30{ }^{\circ} \mathrm{C}$ prior to RNA extraction and RT-qPCR analysis of upregulated and downregulated genes. Shown are the relative expression of CTS1 and CHS2 for each mutant strain compared to the wild type and normalized to ACT1 gene expression. Mean \pm standard error of the mean from three biological replicates, each with three technical replicates. Strains that showed significantly different expression compared to the wild type are indicated. Source data are provided as a Source Data file. Statistical differences were determined using one-way ANOVA with Dunnett's post hoc test for multiple comparisons. CTS1: $\Delta e / m 1$, ${ }^{\star} p=0.0014 ;$ $\Delta \mathrm{elm} 1+$ ELM1, ns: $p=0.35$. CHS2: $\Delta \mathrm{elm} 1, \mathrm{~ns}: p=0.98 ; \Delta \mathrm{elm} 1+E L M 1, \mathrm{~ns}: p=0.24$.

screen. Deletion of ELM1 in AR0382 (Clade I) resulted in a polarized growth phenotype resembling filaments (Fig. 5a). Individual cells remained conjoined at invaginated junctions, forming elongated compartments with similar widths to the wildtype yeast cells. Complementation with the wild-type ELM1 gene restored the wild-type budding yeast morphology (Fig. 4a). Deletion of ELM1 in AR0381 (Clade II) resulted in a similar phenotype as deletion in AR0381 (Clade I), suggesting that its role in regulating polarized growth is conserved across clades (Fig. 5b).

Because filamentous growth has not been shown to be a natural phenotype in $C$. auris, we asked whether the filamentous growth exhibited by $\Delta e l m 1 C$. auris was driven by mechanisms consistent with natural filamentous growth in other fungal species. Maintenance of filamentous growth in other fungal species is achieved through the formation of a Spitzenkörper, a complex of vesicles that coordinates cell wall synthetic enzymes and actin cytoskeleton and related proteins at the apical tip of the growing filament ${ }^{39}$. We observed a structure at the apex of the growing
$\Delta e l m 1$ cells that took up the lipophilic dye FM 4-64, consistent with the formation of a Spitzenkörper (Fig. 5c). This structure appeared to be unique to the filamentous form of AR0382, as no similar polar structure was observed in the budding yeast wildtype or $\Delta e l m 1+E L M 1$ strains (Fig. $5 c$ ).

We next investigated whether the conjoined cell phenotype in AR0382 $\Delta e l m 1$ was associated with alterations in CTS1 or CHS2 regulation like the aggregating mutants. We found the chitinase gene CTS 1 to be significantly upregulated in $\Delta e l m 1$ compared to wild-type AR0382, while the chitin synthase gene CHS2 was not significantly differentially expressed (Fig. 5d). The complemented strain $\Delta e l m 1+E L M 1$ showed no significant variation in the expression of CTS1 or CHS2 compared to wild-type AR0382 (Fig. 5d). Together, these findings implicate ELM1 as a regulator of $C$. auris pseudohyphal growth.

C. auris morphogenic mutants exhibit attenuated virulence and altered antifungal susceptibility. Reports of $C$. auris isolates 
a

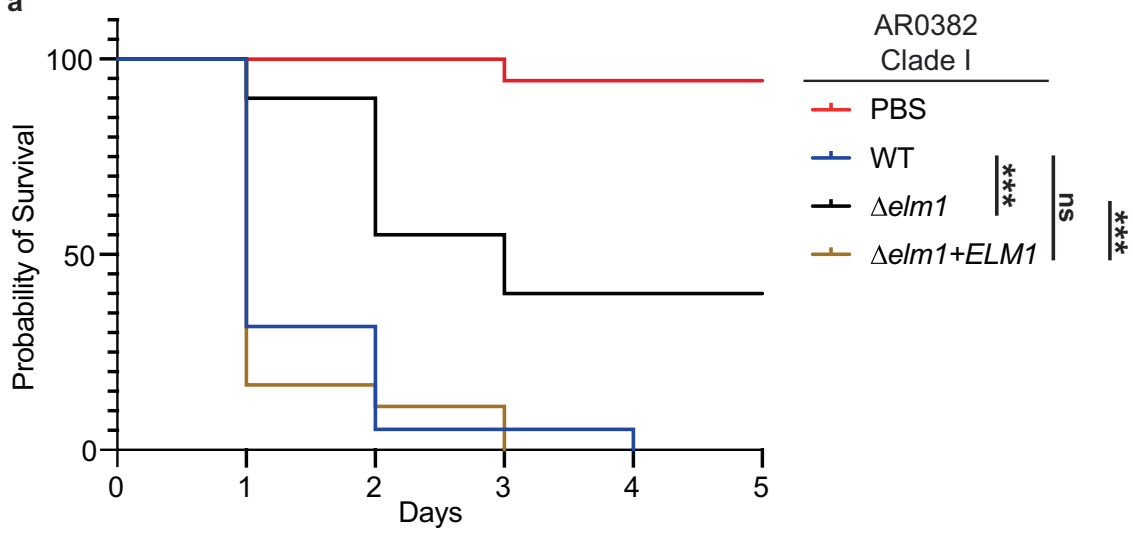

b

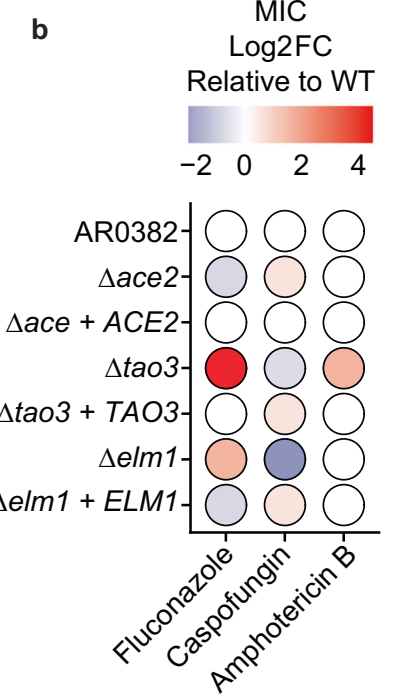

c
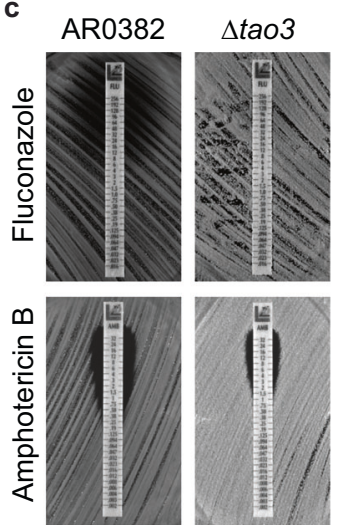

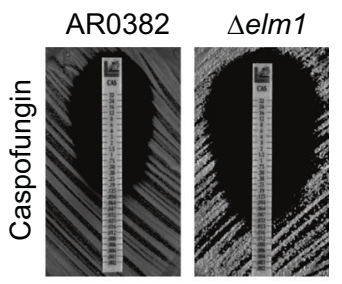

Fig. 6 C. auris morphogenic mutants show attenuated virulence and altered antifungal susceptibility. a Wild-type AR0382 (Clade I), $\triangle$ elm1, and $\Delta$ elm1 + ELM1 strains in the AR0382 background were standardized to an optical density of $\mathrm{OD}_{600}=1.0$ in PBS before inoculating 20 Galleria mellonella larvae per $\mathrm{C}$. auris strain with $50 \mu \mathrm{L}$ of prepared inoculum. Larvae were maintained at $37^{\circ} \mathrm{C}$ and monitored daily for survival for 5 days. Source data are provided as a Source Data file. Statistical differences were determined using a Mantel-Cox log-rank test. WT- $\Delta$ elm1, ${ }^{\star \star \star} p<0.0001 ;$ WT- $\Delta e / m 1+E L M 1$, ns: $p=0.51 ; \Delta e l m 1-\Delta e l m 1+E L M 1,{ }^{\star \star \star} p<0.0001$. b Gradient MIC test strips were used to determine the susceptibility of wild-type AR0382, morphogenic mutants, and complemented strains in the AR0382 background to fluconazole, caspofungin, and amphotericin B. The color and intensity of each point corresponds to the $\log _{2}$ fold change in MIC for each strain relative to the wild type. A complete list of MICs is available in Supplementary Table 1. c MIC test strips with zones of inhibition for mutants with substantially altered antifungal susceptibilities. $\Delta$ tao3 exhibited reduced susceptibility to fluconazole and amphotericin B compared to the wild type. $\Delta$ elm1 exhibited increased susceptibility to caspofungin.

that exhibit aggregating or elongated cell morphologies have largely suggested these morphogenic variants show reduced virulence in infection models compared to budding yeast wildtype isolates $8,11,12$. We therefore investigated the pathogenic potential of the $\Delta a c e 2, \Delta t a o 3$, and $\Delta e l m 1$ mutants in a Galleria mellonella model of infection. The $\Delta$ elm1 strain exhibited the strongest attenuation of virulence compared to the parental AR0382 strain, with mortality rates recapitulating wild-type levels in the complemented $\Delta e l m 1+E L M 1$ strain (Fig. 6a). The $\Delta t a o 3$ mutant did not exhibit significantly attenuated virulence compared to the parental AR0382 ( $p=0.0563$, Log-rank Mantel-Cox test), but the $\triangle a c e 2$ mutant showed a modest decrease in virulence (Supplementary Fig. 5a, b). Complemented strains encoding ACE2 or TAO3 genes showed similar mortality profiles to wild type AR0382 (Supplementary Fig. 5a, b).

We also investigated whether mutations in ACE2, TAO3, or ELM1 were associated with altered antifungal susceptibility profiles. While the $\Delta a c e 2$ mutant did not exhibit a large difference in susceptibility to fluconazole, caspofungin, or amphotericin B compared to wild-type AR0382, the $\Delta$ tao3 and $\Delta e l m 1$ mutants showed differential susceptibility profiles (Fig. 6b). The $\Delta$ tao3 mutant exhibited reduced sensitivity to fluconazole (MIC $>256$ $\mathrm{mg} / \mathrm{L}$ compared to $12 \mathrm{mg} / \mathrm{L}$ for WT AR0382) and amphotericin B (MIC $1 \mathrm{mg} / \mathrm{L}$ compared to $0.38 \mathrm{mg} / \mathrm{L}$ for WT AR0382) (Fig. 6c). The $\Delta e l m 1$ mutant exhibited increased sensitivity to caspofungin (MIC $0.023 \mathrm{mg} / \mathrm{L}$ compared to $0.094 \mathrm{mg} / \mathrm{L}$ for WT AR0382) (Fig. 6c). In general, the complemented strains closely mimicked the susceptibility profile of the wild type (Fig. 6b). A complete list of MICs for each strain against fluconazole, caspofungin, and amphotericin B is available in Supplementary Table 1. Together, these findings demonstrate mutations in C. auris ACE2, TAO3, and ELM1 are associated with altered virulence and antifungal susceptibility profiles in addition to altered morphogenesis.

\section{Discussion}

We have developed approaches to performing facile, costeffective forward and reverse genetic manipulation in C. auris. Using these tools, we identified functional conservation of chitinase regulatory pathways, disruption of which results in 
aggregating, multicellular growth in C. auris. We also uncovered a C. auris filamentous mutant, $\Delta e l m 1$, demonstrating the ability of C. auris to sustain filamentous growth. Our work represents part of a growing global effort to understand the biology of this emerging pathogen by offering alternative methods of improving its genetic tractability. We demonstrated the utility of AtMT for performing insertional mutagenesis in all four major $C$. auris clades without any prior engineering of the C. auris strain. We also demonstrated the ability of a $C$. auris CRISPR-Cas9 expression system to consistently and significantly improve targeted integration of a transformation cassette in representative isolates from all four major C. auris clades. Targeted integration rates were increased to levels at which mutants of interest can readily be identified by PCR or phenotypic screening. While this level of efficiency was associated with approximately $500 \mathrm{bp}$ arms of homology, we successfully performed deletion of TAO3 using a transformation cassette with only $50-70$ bp of homology, albeit with reduced targeted transformation efficiency. Furthermore, we developed a new, codon-optimized G418 selectable marker for use in $C$. auris. Our work, in concert with similar advancements such as successful resistance marker recycling and piggyBac genome-wide transposon mutagenesis in C. auris $8,19,40$, will promote improved accessibility to mechanistic understanding of the genetic machinery in $C$. auris.

From our work, we identified CauACE2 to be a key regulator of morphogenesis. In $S$. cerevisiae, ACE2 daughter cell nuclear localization is regulated by the RAM pathway Kic1-Cbk1 kinase complex $^{34}$. ScTAO3, sometimes called PAG1, physically associates with both $S c K i c 1$ and $S c$ Cbk1 and may mediate activation of Cbk1 by Kic $1^{41,42}$. Disruption of ScTAO3 or downstream ScACE2 results in cellular aggregates and a failure of daughter cells to separate from mother cells during budding ${ }^{38,41,42}$. We observed similar aggregating phenotypes in $\Delta a c e 2$ and $\Delta$ tao 3 mutants in $C$. auris. We therefore propose functional conservation of ACE2 and the RAM regulatory pathway in C. auris. Downstream of this pathway, we identified a putative chitinase, CauCTS1 (B9J08_002761), that was downregulated in $\triangle$ Cauace2 and $\triangle$ Cautao3 compared to the wild type. The sequence of CauCTS1 contains no GPI-anchor signal sequence, and so is likely more closely related functionally to the secreted chitinases ScCTS1 in S. cerevisiae and its functional homolog $\mathrm{CaCHT3}$ in $\mathrm{C}$. albicans than to $\mathrm{CaCHT2}$ in $\mathrm{C}$. albicans ${ }^{43}$. The regulation of CauCTS1 by CauACE2 is consistent with homologous pathways in S. cerevisiae and C. albicans, in which chitin degradation in the primary septum is mediated by the ACE2-regulated $S c C t s 1$ or CaCht3 proteins ${ }^{36,44}$. Interestingly, an experiment performing laboratory evolution of $S$. cerevisiae in a bioreactor resulted in multicellular, fast-sedimenting strains that were associated with mutations in $A C E 2^{35}$. The design of the bioreactors in this example may have provided a selective advantage for multicellular growth due to increased sedimentation rate of cell aggregates compared to planktonic cells. An environmental niche may exist that produces a similar selective pressure against the regulatory network upstream of CTS1 by offering a selective advantage for aggregating cells. Constitutively aggregating strains of $C$. auris have been isolated from clinical samples ${ }^{12-15}$. Based on publicly available RNA-seq data investigating this natural aggregating phenotype, we found that one such aggregating $C$. auris isolate exhibited significantly downregulated expression of CTS1 compared to a non-aggregating counterpart when grown planktonically $\left(\log _{2} \mathrm{FC}=-1.3221, p=6 \mathrm{e}-13\right)^{15}$. This observation is consistent with the mechanism of aggregation observed in the $\Delta a c e 2$ and $\Delta$ tao 3 mutants, though we could not find evidence based on the RNA-seq data that the change in CTS1 regulation of the natural aggregating isolate was directly related to the RAM pathway. Further characterization of the environmental reservoirs for $C$. auris may offer additional insight regarding the selective pressures driving similar phenotypes.

While the role of the serine-threonine kinase ELM1 in regulating polar bud growth and morphogenic differentiation in S. cerevisiae has been long understood, its role in pathogenic fungi is largely unexplored ${ }^{45,46}$. One report demonstrated that deletion of CgELM1 in C. glabrata results in moderately elongated cell growth, though this strain fails to recapitulate the fully pseudohyphal phenotype exhibited by $S$. cerevisiae or C. auris ${ }^{47}$. We observed elongated cells growing in pseudohyphal chains associated with an insertion event near the C-terminus of CauELM1. However, the full $\Delta$ elm1 C. auris strains exhibited a more filamentous cell morphology. This discrepancy in cell morphologies between insertional disruption and clean deletion of elm 1 in $C$. auris may be explained by similar observations in $S$. cerevisiae. In $S$. cerevisiae, deletion of the C-terminal domain (aa 421-640 of 640) of ELM1 results in increased Elm1 kinase activity, suggesting this domain may have autoinhibitory function ${ }^{48}$. This phenotype is associated with pseudohyphal growth with a cell morphology distinct from that demonstrated by $\Delta S c e l m 1^{48}$. The distinct but similarly pseudohyphal phenotypes associated with the C. auris ELM1 insertional mutant encoding a T-DNA insertion 101 base-pairs upstream of the ELM1 C-terminus, but putatively encoding an intact kinase domain, and $\triangle$ Cauelm1 suggests similar ELM1 regulation may exist in C. auris, though the extent to which the ELM1 protein structure is altered for the insertion mutant is unclear. Intriguingly, the filamentous $\Delta e l m 1$ C. auris mutant exhibited a significant increase in the expression of the chitinase gene CTS1 compared to the wild type. This is in contrast to $\Delta e l m 1$ in C. glabrata, which exhibited decreased expression of CgCTS1 compared to wild type ${ }^{47}$. Further characterization of Elm1 in diverse fungal species may yet reveal substantial variation in its function. The role that increased CTS1 expression in $\Delta$ Cauelm1 plays in contributing to filamentous growth is unclear. One report indicated reduced expression of the CTS1 homolog CaCHT3 in hyphal C. albicans compared to $C$. albicans grown in the yeast form ${ }^{49}$. However, total chitinase activity was increased in C. albicans hyphae compared to yeast ${ }^{50}$. Whether C. auris filamentous growth is controlled by a similar chitinase function as $C$. albicans hyphal growth remains to be determined.

In phenotypic analysis, we observed alterations in virulence for the morphogenic mutants, consistent with other published reports ${ }^{8,11,12}$. We were also interested to observe altered antifungal susceptibility profiles associated with these mutants which may hint at larger roles for the genes of interest. In particular, the $\Delta$ tao3 mutant showed markedly reduced susceptibility to fluconazole and amphotericin $B$ that appeared to be independent of $A C E 2$, as the $\triangle a c e 2$ mutant did not show the same altered susceptibilities. The resistance to fluconazole conferred by deletion of TAO3 may indicate the C. auris RAM pathway upstream of ACE2 has divergent regulatory roles from the same pathway in $C$. albicans, mutation of which confers hypersensitivity to fluconazole ${ }^{51}$. Similarly, C. albicans ace $2 \Delta /$ ace $2 \Delta$ cells exhibit increased resistance to fluconazole that was not observed for $C$. auris $\Delta$ ace 2 cells $^{52}$. While the full mechanistic contribution of the C. auris RAM pathway to antifungal susceptibility remains unclear, these observations highlight the possibility of regulatory network rewiring in C. auris and the importance of specifically investigating how $C$. auris has adapted regulatory pathways that may have well-established mechanisms in related model fungal species.

In sum, our work demonstrates an accessible approach to genetic engineering of $C$. auris, facilitating further understanding of the biology of this emerging pathogen. Using new forward and reverse genetic approaches, we characterized conserved and divergent key regulators of morphogenesis, virulence, and antifungal resistance in C. auris. 


\section{Methods}

Strains and growth conditions. A list of C. auris and A. tumefaciens strains used in this study are listed in Supplementary Table 2. Unless specified otherwise, $C$. auris cells were grown at $30^{\circ} \mathrm{C}$ in YPD liquid media ( $1 \%$ yeast extract, $2 \%$ peptone, $2 \%$ dextrose) with constant agitation. All strains were maintained in frozen stocks of $25 \%$ glycerol at $-80^{\circ} \mathrm{C}$.

Plasmids. A list of all plasmids used in this study is included in Supplementary Table 3. Constructs and sequences for pTO128, pTO135, pTO136, and pTO149 are available through Addgene (Watertown, MA, USA) under catalog \#171105, \#171103, \#171104, and \#177277.

A list of all primers used in this study is included in Supplementary Table 4.

pTO128. An Agrobacterium Ti-plasmid was constructed to include the CaNAT1 nourseothricin resistance cassette ${ }^{53}$ in the pPZP-NEO1 backbone ${ }^{54}$. The CaNAT1 cassette was excised at the SacI and SalI restriction sites from pLC49 ${ }^{55}$ and ligated between the SacI and SalI restriction sites of pPZP-NEO1, replacing the G418 resistance cassette with CaNAT1 to form pTO128 (pPZP-NATca). pTO128 was subsequently electroporated into A. tumefaciens strain EHA $105^{56}$ using a Bio-Rad MicroPulser Electroporator.

All other transformation cassettes were maintained in the multiple cloning site of the pUC19 cloning vector ${ }^{57}$ and assembled from fragments as described below using the NEBuilder HIFI DNA Assembly master mix (NEB \#E2621) according to the manufacturer's instructions.

pTO135. To assemble pTO135 (pCauCas9), the Cas9 expression cassette minus the promoter sequence was PCR amplified from pLC963 ${ }^{58}$ using primers oTO114oTO115. A promoter region consisting of $1000 \mathrm{bp}$ upstream of the C. auris ENO1 gene (B9J08 000274) was PCR amplified from genomic DNA isolated from $C$. auris strain AR0387 using primers oTO112-oTO113. The pUC19 vector backbone was amplified using primers oTO116-oTO117.

pTO136. To assemble pTO136 (pCausgRNA), a promoter region consisting of 901 bp upstream of the C. auris ADH1 gene (B9J08_004331) was PCR amplified using primers oTO118-oTO119 and assembled along with a synthesized DNA fragment (Genscript, Piscataway, NJ, USA) containing sequence from C. auris tRNA-Ala (B9J08_003096), a 20-bp gRNA sequence targeting the ENO1 locus, a tracrRNA sequence, and an HDV ribozyme ${ }^{31}$. The pUC19 vector backbone was amplified using primers oTO120-oTO121.

pTO137. To assemble pTO137, containing the ENO1-RFP reporter cassette, the RFP construct was PCR amplified from pLC1047 ${ }^{59}$ using primers oTO124oTO125; a terminator sequence consisting of $933 \mathrm{bp}$ downstream of the $C$. auris $A D H 1$ gene was PCR amplified from $C$. auris AR0387 genomic DNA using primers oTO126-oTO127; the CaNAT1 expression cassette including TEF promoter and terminator sequence was amplified from pLC49 using primers oTO128-oTO129; flanking regions containing homology to $492 \mathrm{bp}$ at the C-terminal end of the $C$. auris ENO1 gene minus the stop codon and $557 \mathrm{bp}$ immediately $3^{\prime}$ of the $C$. auris ENO1 gene were amplified from genomic DNA isolated from $C$. auris strain AR0387 genomic DNA using primers oTO122-oTO123 and oTO130-oTO131 respectively; the pUC19 vector backbone was amplified using primers oTO132oTO133.

pTO154. To assemble pTO154 (pELM1::NAT), $501 \mathrm{bp}$ immediately $5^{\prime}$ of ELM1 (B9J08_002849) and 502 bp immediately $3^{\prime}$ of ELM1 were amplified from AR0387 genomic DNA using primers oTO317-oTO318 and oTO321-oTO337 respectively; the CaNAT1 expression cassette was amplified from pLC49 using primers oTO319oTO320; the pUC19 vector backbone was amplified using primers oTO323oTO324.

pTO155. To assemble pTO155 (pACE2::NAT), $500 \mathrm{bp}$ immediately $5^{\prime}$ of ACE2 (B9J08 000468) and $498 \mathrm{bp}$ immediately $3^{\prime}$ of ACE2 were amplified from AR0387 genomic DNA using primers oTO325-oTO326 and oTO329-oTO330 respectively; the CaNAT1 expression cassette was amplified from pLC49 using primers oTO327oTO328; the pUC19 vector backbone was amplified using primers oTO331oTO332.

pTO149. pTO149 was assembled to maintain the NEO (G418) resistance cassette. A codon-optimized NEO resistance gene was synthesized with every CUG codon replaced by the synonymous CUC leucine codon. This was assembled into pTO137 in place of the NAT resistance cassette, the backbone amplified with oTO272oTO273. The codon-optimized NEO resistance gene was amplified including the $T E F$ promoter and terminator sequence for assembly into complementation cassettes.

pTO169. To assemble pTO169 (pace2 + ACE2), the full-length ACE2 gene along with 1001 bp upstream was amplified from AR0382 genomic DNA using oTO566oTO567, the $A D H 1$ terminator sequence and the NEO resistance cassette were amplified from pTO149 with oTO568-oTO569, 832 bp downstream of ACE2 was amplified from AR0382 genomic DNA using oTO570-oTO571, and the pUC19 backbone was amplified using oTO564-oTO565.

pTO174. To assemble pTO174 (ptao3 + TAO3), the full-length TAO3 gene along with 869 bp upstream was amplified from AR0382 genomic DNA using oTO584 oTO585, the $A D H 1$ terminator sequence and the $N E O$ resistance cassette were amplified from pTO149 with oTO586-oTO587, 503 bp downstream of TAO3 was amplified from AR0382 genomic DNA using oTO588-oTO589, and the pUC19 backbone was amplified using oTO582-oTO583.

pTO175. To assemble pTO175 (pelm1 + ELM1), the full-length ELM1 gene along with 997 bp upstream was amplified from AR0382 genomic DNA using oTO592oTO593, the ADH1 terminator sequence and the NEO resistance cassette were amplified from pTO149 with oTO594-oTO595, 582 bp downstream of ELM1 was amplified from AR0382 genomic DNA using oTO596-oTO597, and the pUC19 backbone was amplified using oTO590-oTO591.

All C. auris genomic sequence data were obtained from the C. auris B8441 reference genome on fungidb.org ${ }^{60}$. All plasmid assemblies were verified by restriction digest and sanger sequencing.

Agrobacterium tumefaciens-mediated transformation (AtMT). AtMT was performed as previously described with minor modifications ${ }^{61}$. Briefly, A. tumefaciens strain EHA 105 harboring the pTO128 (pPZP-NATca) plasmid was cultured overnight at $30^{\circ} \mathrm{C}$ in liquid Luria-Bertani (LB) media containing kanamycin. $A$. tumefaciens cells were harvested by centrifugation, washed once with sterile, ultrapure water, then resuspended at a final $\mathrm{OD}_{600}$ of 0.15 in liquid Induction Medium (IM) supplemented with $100 \mu \mathrm{M}$ Acetosyringone $3^{\prime}, 5^{\prime}$-dimethoxy-4hydroxyacetophenone (AS) 28 and incubated at room temperature for $6 \mathrm{~h}$ with constant agitation. Recipient $C$. auris cells were harvested from an overnight culture grown at $30^{\circ} \mathrm{C}$ in YPD by centrifugation then resuspended in sterile, ultrapure water at a final $\mathrm{OD}_{600}$ of 1.0. Equal volumes of prepared A. tumefaciens and $C$. auris were combined and the mixed culture was incubated on IM with AS agar at $23^{\circ} \mathrm{C}$ for 4 days. Cells were then harvested into liquid YPD, washed three times with fresh YPD, then spread-plated onto YPD agar containing $200 \mu \mathrm{g} / \mathrm{mL}$ nourseothricin and $200 \mu \mathrm{g} / \mathrm{mL}$ cefotaxime. Plates were incubated at $30^{\circ} \mathrm{C}$ for 2 days. Transformation efficiency was determined by dividing the total number of recovered $C$. auris CFU by the total input number of $C$. auris cells. To identify morphogenic mutants, colonies were screened visually for those exhibiting a wrinkled colony morphology then confirmed to exhibit aggregating or filamentous phenotypes using light microscopy.

Genomic DNA isolation. Genomic DNA was isolated from C. auris morphological mutants to be used for downstream sequencing and insertion site mapping using a phenol-chloroform extraction. Briefly, cells were incubated overnight at $30^{\circ} \mathrm{C}$ in liquid YPD then harvested by centrifugation and resuspended in breaking buffer (2\% (v/v) Triton X-100, 1\% (w/v) SDS, $100 \mathrm{mM} \mathrm{NaCl,} 10 \mathrm{mM}$ Tris-Cl, $1 \mathrm{mM}$ EDTA). DNA was extracted by bead beating into PCA then extracted into chloroform. Following precipitation by ethanol, extracted DNA was resuspended in TE buffer and treated with RNase A. Genomic DNA quality was assessed by $1 \%$ agarose gel electrophoresis.

ATMT transgene mapping. Mapping of T-DNA insertion sites was performed similarly to methods previously described ${ }^{29}$. Genomic DNA isolated from six morphogenic mutants was collected and divided into two pools, each containing equal amounts by mass of genomic DNA from three individual mutants. Library preparation, quality control, and whole-genome sequencing were performed by Microbial Genome Sequencing Center (MIGS, Pittsburg, PA, USA). Library preparation was performed based on the Illumina Nextera kit and sequencing performed on the Nextseq 550 platform, generating $150 \mathrm{bp}$ paired-end reads for each pool. Sequencing data were analyzed using the Galaxy web platform public server at usegalaxy.org 62 . Read quality was assessed using FASTQC and reads were trimmed using CutAdapt ${ }^{63}$ with a Phred quality cutoff of 20. A linearized vector reference sequence of pTO128 (pPZP-Natca) was generated from the circular vector sequence and $150 \mathrm{bp}$ of sequence from the opposite border was added to each border of the linearized sequence. Reads were mapped to the linear pTO128 (pPZP-NATca) reference sequence using the Burrows-Wheeler Aligner with maximum exact matches (BWA-MEM) ${ }^{64}$ configured with default parameters except for minimum seed length $=50$ and band width $=2$. Mapped reads were visualized using $\mathrm{IGV}^{62}$ and sorted based on position and sequences that extended beyond the left and right boundaries of the tDNA was extracted. Consensus sequences of the extracted reads were mapped to the C. auris B8441 reference genome (GCA_002759435.2) using NCBI Blast. Primers specific to each identified insertion site were designed: oTO310 and oTO340 for B9J08_002252, oTO311 and oTO344 for B9J08_003879, oTO312 and oTO342 for B9J08_002849, oTO313 and oTO338 for B9J08_000181, oTO314 and oTO339 for B9J08_000468, oTO315 and oTO341 for B9J08 002667, and oTO316 and oTO343 for B9J08_002954. These were used to amplify the identified insertion regions in conjunction with T-DNA specific primers oTO6 and oTO90 using the genomic DNA from each of the six 
mutants as templates. Individual insertions were attributed to individual mutants based on amplicon length. Amplicons containing T-DNA insertions were Sanger sequenced to generate insertion maps for each mutant.

C. auris transformation. Transformation of C. auris was performed as described previously, with minor modifications ${ }^{18}$. To generate ENO1-RFP strains, linear transformation cassettes encoding Cas9, sgRNA, and the RFP repair cassette were PCR amplified from pTO135, pTO136, and pTO137, respectively, using primers oTO18-oTO19. To generate $\triangle a c e 2, \Delta e l m 1, \Delta a c e 2+A C E 2, \Delta t a o 3+T A O 3$, and $\Delta e l m 1+E L M 1$ strains, a linear Cas9 cassette was amplified from pTO135 using primers oTO18-oTO19, linear repair cassettes were amplified from pTO155 for ACE2::NAT, pTO154 for ELM1::NAT, pTO169 for $\triangle a c e 2+A C E 2$, pTO174 for $\triangle t a 03+T A O 3$, and pTO175 for $\triangle e l m 1+E L M 1$ using primers oTO18-oTO19. To generate $\Delta t a o 3$, a linear repair cassette incorporating $50-70 \mathrm{bp}$ homology to either end of the target gene flanking the NAT cassette was amplified from pTO137 using primers oTO353-oTO354. Linear sgRNA cassettes were amplified from pTO136 using fusion PCR as described previously to replace the gRNA sequence with gRNA targeting each gene for deletion ${ }^{20}$. Fusion fragments were amplified using primers oTO333-oTO225 and oTO224-oTO334 to target ELM1, oTO335-oTO225 and oTO224-oTO336 to target ACE2, oTO356-oTO224 and oTO355-oTO225 to target TAO3, and oTO224-oTO519 and oTO225-oTO518 to target NAT. Each pair of fragments with overlapping sequences were spliced on extension using oTO18oTO19. PCR products were purified with a Zymo DNA Clean \& Concentrator kit (Cat no. D4034, Zymo Research) according to the manufacturer's instructions. C. auris cells were incubated overnight at $30^{\circ} \mathrm{C}$ in YPD to exponential phase, not exceeding $\mathrm{OD}_{600}$ of 2.2. Cells were harvested by centrifugation and resuspended in TE buffer with $100 \mathrm{mM}$ lithium acetate then incubated with constant shaking at $30^{\circ} \mathrm{C}$ for $1 \mathrm{~h}$. DTT was added to the cells at a final concentration of $25 \mathrm{mM}$ and incubation was continued for $30 \mathrm{~min}$ at $30^{\circ} \mathrm{C}$ with constant shaking. The cells were harvested by centrifugation; washed once with ice-cold, sterile, ultrapure water; washed once with ice-cold $1 \mathrm{M}$ sorbitol; then resuspended in ice-cold $1 \mathrm{M}$ sorbitol. $40 \mu \mathrm{L}$ of competent cells was added to a pre-chilled 2-mm-gap electro-cuvette along with $1 \mu \mathrm{g}$ each of the PCR-amplified linear transformation cassettes encoding Cas9, sgRNA, and the repair cassette. Alternatively, to compare targeted integration efficiency, an equal volume of Zymo elution buffer was added instead of Cas9 or sgRNA cassettes. Cells were electroporated using a Bio-Rad MicroPulser Electroporator set to the programmed P. pastoris (PIC) protocol ( $2.0 \mathrm{kV}, 1$ pulse), recovered in $1 \mathrm{M}$ sorbitol, then resuspended in YPD and allowed $2 \mathrm{~h}$ of outgrowth at $30^{\circ} \mathrm{C}$ with shaking. The cells were then spread-plated on YPD with $200 \mu \mathrm{g} / \mathrm{mL}$ nourseothricin and incubated at $30^{\circ} \mathrm{C}$ or $1000 \mu \mathrm{g} / \mathrm{mL} \mathrm{G} 418$ and incubated at $23^{\circ} \mathrm{C}$. Mutant strains were confirmed with PCR and Sanger sequencing and were confirmed to not exhibit stable integration of the CAS9 cassette using CAS9-specific PCR primers oTO514-oTO515.

To estimate the efficiency of targeted RFP integration among transformant colonies, transformation plates were imaged using a Typhoon FLA 9500 Bioimager fitted with a $532 \mathrm{~nm}$ filter. Fluorescent images were visualized using Fiji Software ${ }^{63}$. An intensity threshold was set to identify transformant colonies exhibiting fluorescence. Five representative fluorescent colonies and five representative nonfluorescent colonies from transformations performed in AR0387 were spotted onto YPD agar and grown at $30^{\circ} \mathrm{C}$ for 2 days. A sample of the colony growth was collected from each colony and suspended in $15 \mu \mathrm{L}$ water. An aliquot of this suspension was used as a template in PCR reactions with primers overlapping the junction of the predicted ENO1-RFP insertion site or a genomic region upstream of the junction present in the wild-type locus. Colony PCR was performed using Phire Plant Direct PCR Master Mix (F160S; Thermo Fisher Scientific) according to the manufacturer's instructions. The proportion of transformant colonies with targeted integration was determined by dividing the number of colonies exhibiting fluorescence by the total number of transformant colonies.

Live cell microscopy. Cells were grown to mid-exponential phase at $30^{\circ} \mathrm{C}$ in YPD and pelleted by centrifugation for $1 \mathrm{~min}$ at $4000 \mathrm{rpm}(1500 \times g)$ then resuspended in PBS. $5 \mu \mathrm{L}$ cell suspension was combined with $1 \mu \mathrm{L}$ of $0.1 \mathrm{~g} / \mathrm{L}$ Calcofluor White stain and applied to a glass microscope slide. Alternatively, overnight cultures were prepared for each isolate and wild-type strain in yeast extract peptone dextrose (YPD) at $30^{\circ} \mathrm{C}$, with rotation, and then subcultured to mid-log phase before washing twice in $1 \times$ PBS and staining with FM4-64 (BioTracker 640 Red C2(FM4-64) Synaptic Dye, Millipore Sigma) at $10 \mu \mathrm{M}$ for $10 \mathrm{~min}$. Cells were visualized using an Olympus IX70 Epifluorescent Microscope fitted with a Hamamatsu C11440 camera and taken with Olympus CellSens v. 3.2 software.

Stereomicroscopy. C. auris cells were grown on YPD agar at $30^{\circ} \mathrm{C}$ for $2-7$ days to form colonies. Colonies were visualized using a Leica KL300 LED stereomicroscope.

RNA extraction. RNA extraction was performed as described previously ${ }^{64}$. Briefly, cells were grown to mid-exponential phase at $30^{\circ} \mathrm{C}$ in YPD and harvested by centrifugation. Cells were washed in PBS, then centrifuged and all liquid removed. Dry cell pellets were frozen on dry ice then stored at $-80^{\circ} \mathrm{C}$ overnight. Cell pellets were thawed and resuspended in $100 \mu \mathrm{L}$ FE Buffer (98\% formamide, $0.01 \mathrm{M}$ EDTA) at room temperature. $50 \mu \mathrm{L}$ of $500 \mu \mathrm{m}$ RNAse-free glass beads was added and the cell suspension was ground in three cycles of $30 \mathrm{~s}$ using a BioSpec MiniBeadbeater-16 (Biospec Products Inc., Bartlesville, OK, USA). The cell lysate was centrifuged to remove cell debris and the crude RNA extract collected from the supernatant. The extract was DNAse-treated and purified using a Qiagen RNeasy mini kit (ref. 74104, Qiagen) as per the manufacturer's instructions. RNA integrity was confirmed through agarose gel electrophoresis using the bleach gel method ${ }^{65}$.

RT-qPCR. cDNA was synthesized from isolated RNA using the AffinityScript qPCR cDNA Synthesis Kit (ref. 600559, Agilent Technologies) according to the manufacturer's instructions and used as a template for qPCR. qPCR was performed in three biological replicates, each with three technical replicates using a Bio-Rad CFXConnect Real Time System. Fold changes were calculated using the doubledelta CT method with expression normalized to that of $A C T 1$ and compared to wild type. Amplification was measured for ACT1 using primers oTO359-oTO360, for CHS2 using primers oTO361-oTO362, and for CTS1 using primers oTO363oTO364.

Co-expression genetic interaction analysis. The C. albicans ortholog of B9J08_002252 was identified through orthology on the Candida Genome Database as C7_00260C_A. This was used as a query in CalCEN and the top 50 most coexpressed neighbors were identified. This set was then examined for putative function through GO term enrichment in the Candida Genome database. The network was visualized using Cytoscape.

Galleria mellonella infections. Infections were performed as previously described $^{66}$, with minor modifications. Briefly, G. mellonella larvae were purchased from speedyworm.com and maintained in sawdust at room temperature. Overnight C. auris cultures were prepared for each isolate and wild-type strain in yeast extract peptone dextrose (YPD) at $30^{\circ} \mathrm{C}$, with rotation. We were unable to standardize inoculum by direct cell count between aggregating and non-aggregating strains, so we standardized each inoculum to a consistent optical density. Cells were washed twice in PBS and diluted to an OD600 of 1 in $1 \times$ PBS, and $50 \mu \mathrm{L}$ was inoculated into the larvae using an exel veterinary U-40 diabetic syringe $(0.5 \mathrm{CC} \times 29 \mathrm{G} \times 1 / 2)$. Twenty larvae were infected per $C$. auris strain. After injection, larvae were maintained at $37^{\circ} \mathrm{C}$ and monitored daily for survival. Virulence was analyzed using Kaplan-Meier survival curves in GraphPad Prism (version 9).

Antifungal susceptibility assays. C. auris colonies were suspended in PBS to $\mathrm{OD}_{600}=1.0$. A sterile cotton applicator was saturated with the cell suspension and used to inoculate the entire surface of a YPD plate three times, rotating the plate approximately $60^{\circ}$ each time. The surface of the agar was allowed to dry at room temperature. MIC test strips containing $0.016-256 \mathrm{mg} / \mathrm{L}$ Fluconazole (Liofilchem REF 921471), 0.002-32 mg/L Caspofungin (Liofilchem REF 921541), or 0.002-32 mg/L Amphotericin B (Liofilchem REF 921531) were placed onto the surface of the agar. Plates were incubated inverted at $37^{\circ} \mathrm{C}$ for $24 \mathrm{~h}$ and MICs were determined at the intersection between the zone of inhibition and the MIC test strip gradient.

Statistics and reproducibility. Statistical analyses were carried out using R statistical software (version 4.3) or GraphPad Prism (version 9). Data are presented as means \pm standard error of means from biological replicates. Except where otherwise specified, each experiment was performed in at least three independent biological replicates yielding similar results. Statistical significance among different groups was calculated using one-way ANOVA, with Tukey's or Dunnett's post hoc tests for multiple comparisons, chi-square test, or Mantel-Cox log-rank test. ${ }^{*} p \leq 0.05 ;{ }^{* *} p \leq 0.01 ;{ }^{* * *} p \leq 0.001$; n.s., $p>0.05$.

Reporting summary. Further information on research design is available in the Nature Research Reporting Summary linked to this article.

\section{Data availability}

Data from Illumina sequences used to identify transgene insertion sites are available in the NCBI SRA under BioProject accession number PRJNA722500. Gene sequences for mapping and designing constructs were retrieved from the B8441 genome assembly (NCBI GCA_002759435.2) through fungidb.org. Source data are provided with this paper.

Received: 7 May 2021; Accepted: 29 November 2021; Published online: 10 December 2021

\section{References}

1. Chakrabarti, A. \& Sood, P. On the emergence, spread and resistance of Candida auris: host, pathogen and environmental tipping points. J. Med. Microbiol. https://doi.org/10.1099/jmm.0.001318 (2021). 
2. Chow, N. A. et al. Tracing the evolutionary history and global expansion of Candida auris using population genomic analyses. MBio 11, e03364-19 (2020).

3. Chow, N. A. et al. Potential fifth clade of Candida auris, Iran, 2018. Emerg. Infect. Dis. 25, 1780-1781 (2019).

4. Forgács, L. et al. Comparison of in vivo pathogenicity of four Candida auris clades in a neutropenic bloodstream infection murine model. Emerg. Microbes Infect. 9, 1160-1169 (2020).

5. Huang, X. et al. Murine model of colonization with fungal pathogen Candida auris to explore skin tropism, host risk factors and therapeutic strategies. Cell Host Microbe https://doi.org/10.1016/j.chom.2020.12.002 (2020).

6. Kim, S. H. et al. Genetic analysis of Candida auris implicates Hsp90 in morphogenesis and azole tolerance and Cdr1 in azole resistance. MBio 10, e02529-18 (2019).

7. Bravo Ruiz, G., Ross, Z. K., Gow, N. A. R. \& Lorenz, A. Pseudohyphal growth of the emerging pathogen Candida auris is triggered by genotoxic stress through the S phase checkpoint. mSphere 5, e00151-20 (2020).

8. Gao, J. et al. LncRNA DINOR is a virulence factor and global regulator of stress responses in Candida auris. Nat. Microbiol. https://doi.org/10.1038/ s41564-021-00915-x (2021).

9. Wang, X. et al. The first isolate of Candida auris in China: clinical and biological aspects. Emerg. Microbes Infect. 7, 93 (2018).

10. Yue, H. et al. Filamentation in Candida auris, an emerging fungal pathogen of humans: passage through the mammalian body induces a heritable phenotypic switch. Emerg. Microbes Infect. 7, 188 (2018).

11. Fan, S. et al. Filamentous growth is a general feature of Candida auris clinical isolates. Med. Mycol. https://doi.org/10.1093/mmy/myaa116 (2021).

12. Borman, A. M., Szekely, A. \& Johnson, E. M. Comparative pathogenicity of United Kingdom isolates of the emerging pathogen Candida auris and other key pathogenic Candida species. mSphere 1, e00189-16 (2016).

13. Short, B. et al. Candida auris exhibits resilient biofilm characteristics in vitro: implications for environmental persistence. J. Hosp. Infect. 103, 92-96 (2019).

14. Szekely, A., Borman, A. M. \& Jphnson, E. M. Candida auris isolates of the Southern Asian and South African lineages exhibit different phenotypic and antifungal susceptibility profiles in vitro. J. Clin. Microbiol. 57, e02055-18 (2019).

15. Brown, J. L. et al. Candida auris phenotypic heterogeneity determines pathogenicity in vitro. mSphere 5, e00371-20 (2020).

16. Sherry, L. et al. Biofilm-forming capability of highly virulent, multidrugresistant Candida auris. Emerg. Infect. Dis. 23, 328-331 (2017).

17. Mayr, E.-M., Ramírez-Zavala, B., Krüger, I. \& Morschhäuser, J. A zinc cluster transcription factor contributes to the intrinsic fluconazole resistance of Candida auris. $m$ Sphere 5, e00279-20 (2020).

18. Grahl, N., Demers, E. G., Crocker, A. W. \& Hogan, D. A. Use of RNA -protein complexes for genome editing in non-albicans Candida species. mSphere 2, e00218-17 (2017).

19. Rybak, J. M. et al. Abrogation of triazole resistance upon deletion of CDR1 in a clinical isolate of Candida auris. Antimicrob. Agents Chemother. 63, e0005719 (2019).

20. Min, K., Ichikawa, Y., Woolford, C. A. \& Mitchell, A. P. Candida albicans gene deletion with a transient CRISPR-Cas9 system. mSphere 1, e00130-16 (2016).

21. Fan, Y. \& Lin, X. Multiple applications of a transient CRISPR-Cas9 coupled with electroporation (TRACE) system in the Cryptococcus neoformans species complex. Genetics 208, 1357-1372 (2018).

22. Soltani, J., van Heusden, G. P. H. \& Hooykaas, P. J. J. in Agrobacterium: From Biology to Biotechnology (eds Tzfira, T. \& Citovsky, V.) 649-675 (Springer, 2008).

23. Cleene, M. D., De Cleene, M. \& De Ley, J. The host range of crown gall. Botanical Rev. 42, 389-466 (1976).

24. Michielse, C. B., Hooykaas, P. J. J., van den Hondel, C. A. M. J. J. \& Ram, A. F. J. Agrobacterium-mediated transformation as a tool for functional genomics in fungi. Curr. Genet. 48, 1-17 (2005).

25. Hooykaas, P. J. J. et al. Agrobacterium-mediated transformation of yeast and fungi. Curr. Top. Microbiol. Immunol. 418, 349-374 (2018).

26. Lutgring, J. D. et al. FDA-CDC antimicrobial resistance isolate bank: a publicly available resource to support research, development, and regulatory requirements. J. Clin. Microbiol. 56, e01415-e01417 (2018).

27. McClelland, C. M., Chang, Y. C. \& Kwon-Chung, K. J. High frequency transformation of Cryptococcus neoformans and Cryptococcus gattii by Agrobacterium tumefaciens. Fungal Genet. Biol. 42, 904-913 (2005).

28. Bundock, P., den Dulk-Ras, A., Beijersbergen, A. \& Hooykaas, P. J. Transkingdom T-DNA transfer from Agrobacterium tumefaciens to Saccharomyces cerevisiae. EMBO J. 14, 3206-3214 (1995).

29. Park, D. et al. A bioinformatics approach for identifying transgene insertion sites using whole genome sequencing data. BMC Biotechnol. 17, 67 (2017).

30. O'Meara, T. R. \& O'Meara, M. J. DeORFanizing Candida albicans genes using coexpression. mSphere 6, e01245-20 (2021)

31. Ng, H. \& Dean, N. Dramatic improvement of CRISPR/Cas9 editing in Candida albicans by increased single guide RNA expression. mSphere 2, e00385-16 (2017).
32. Schiffer, S., Rösch, S. \& Marchfelder, A. Assigning a function to a conserved group of proteins: the tRNA 3'-processing enzymes. EMBO J. 21, 2769-2777 (2002)

33. Gao, Y. \& Zhao, Y. Self-processing of ribozyme-flanked RNAs into guide RNAs in vitro and in vivo for CRISPR-mediated genome editing. J. Integr. Plant Biol. 56, 343-349 (2014).

34. Saputo, S., Chabrier-Rosello, Y., Luca, F. C., Kumar, A. \& Krysan, D. J. The RAM network in pathogenic fungi. Eukaryot. Cell 11, 708-717 (2012).

35. Oud, B. et al. Genome duplication and mutations in ACE2 cause multicellular, fast-sedimenting phenotypes in evolved Saccharomyces cerevisiae. Proc. Natl Acad. Sci. USA 110, E4223-E4231 (2013)

36. Kelly, M. T. et al. The Candida albicans CaACE2 gene affects morphogenesis, adherence and virulence. Mol. Microbiol. 53, 969-983 (2004).

37. Calderón-Noreña, D. M. et al. A single nucleotide polymorphism uncovers a novel function for the transcription factor Ace2 during Candida albicans hyphal development. PLoS Genet. 11, e1005152 (2015).

38. King, L. \& Butler, G. Ace2p, a regulator of CTS1 (chitinase) expression, affects pseudohyphal production in Saccharomyces cerevisiae. Curr. Genet. 34, 183-191 (1998).

39. Riquelme, M. \& Sánchez-León, E. The Spitzenkörper: a choreographer of fungal growth and morphogenesis. Curr. Opin. Microbiol. 20, 27-33 (2014)

40. Rybak, J. M. et al. Mutations in TAC1B: a novel genetic determinant of clinical fluconazole resistance in Candida auris. mBio 11, e00365-20 (2020).

41. Nelson, B. et al. RAM: a conserved signaling network that regulates Ace2p transcriptional activity and polarized morphogenesis. Mol. Biol. Cell 14, 3782-3803 (2003)

42. Du, L.-L. \& Novick, P. Paglp, a novel protein associated with protein kinase Cbk1p, is required for cell morphogenesis and proliferation in Saccharomyces cerevisiae. $M B o C$ 13, 503-514 (2002).

43. Dünkler, A., Walther, A., Specht, C. A. \& Wendland, J. Candida albicans CHT3 encodes the functional homolog of the Cts1 chitinase of Saccharomyces cerevisiae. Fungal Genet. Biol. 42, 935-947 (2005).

44. Kuranda, M. J. \& Robbins, P. W. Chitinase is required for cell separation during growth of Saccharomyces cerevisiae. J. Biol. Chem. 266, 19758-19767 (1991).

45. Koehler, C. M. \& Myers, A. M. Serine-threonine protein kinase activity of Elm1p, a regulator of morphologic differentiation in Saccharomyces cerevisiae. FEBS Lett. 408, 109-114 (1997).

46. Sreenivasan, A. \& Kellogg, D. The elm1 kinase functions in a mitotic signaling network in budding yeast. Mol. Cell. Biol. 19, 7983-7994 (1999).

47. Ito, Y. et al. Roles of Elm1 in antifungal susceptibility and virulence in Candida glabrata. Sci. Rep. 10, 9789 (2020).

48. Sutherland, C. M. et al. Elm1p is one of three upstream kinases for the Saccharomyces cerevisiae SNF1 complex. Curr. Biol. 13, 1299-1305 (2003).

49. McCreath, K. J., Specht, C. A. \& Robbins, P. W. Molecular cloning and characterization of chitinase genes from Candida albicans. Proc. Natl Acad. Sci. USA 92, 2544-2548 (1995).

50. Selvaggini, S., Munro, C. A., Paschoud, S., Sanglard, D. \& Gow, N. A. R Independent regulation of chitin synthase and chitinase activity in Candida albicans and Saccharomyces cerevisiae. Microbiology 150, 921-928 (2004).

51. Song, Y. et al. Role of the RAM network in cell polarity and hyphal morphogenesis in Candida albicans. Mol. Biol. Cell 19, 5456-5477 (2008),

52. Homann, O. R., Dea, J., Noble, S. M. \& Johnson, A. D. A phenotypic profile of the Candida albicans regulatory network. PLoS Genet. 5, e1000783 (2009).

53. Shen, J., Guo, W. \& Köhler, J. R. CaNAT1, a heterologous dominant selectable marker for transformation of Candida albicans and other pathogenic Candida species. Infect. Immun. 73, 1239-1242 (2005).

54. Walton, F. J., Idnurm, A. \& Heitman, J. Novel gene functions required for melanization of the human pathogen Cryptococcus neoformans. Mol. Microbiol. 57, 1381-1396 (2005).

55. Cowen, L. E. et al. Harnessing Hsp90 function as a powerful, broadly effective therapeutic strategy for fungal infectious disease. Proc. Natl Acad. Sci. USA 106, 2818-2823 (2009).

56. Hood, E. E., Gelvin, S. B., Melchers, L. S. \& Hoekema, A. New Agrobacterium helper plasmids for gene transfer to plants. Transgenic Res. 2, 208-218 (1993).

57. Norrander, J., Kempe, T. \& Messing, J. Construction of improved M13 vectors using oligodeoxynucleotide-directed mutagenesis. Gene 26, 101-106 (1983).

58. Veri, A. O. et al. Tuning Hsfl levels drives distinct fungal morphogenetic programs with depletion impairing Hsp90 function and overexpression expanding the target space. PLoS Genet. 14, e1007270 (2018).

59. O'Meara, T. R. et al. Global proteomic analyses define an environmentally contingent Hsp90 interactome and reveal chaperone-dependent regulation of stress granule proteins and the R2TP complex in a fungal pathogen. PLoS Biol. 17, e3000358 (2019).

60. Basenko, E. Y. et al. FungiDB: An Integrated Bioinformatic Resource for Fungi and Oomycetes. J. Fungi 4, 39 (2018).

61. Esher, S. K., Granek, J. A. \& Alspaugh, J. A. Rapid mapping of insertional mutations to probe cell wall regulation in Cryptococcus neoformans. Fungal Genet. Biol. 82, 9-21 (2015). 
62. Robinson, J. T. et al. Integrative genomics viewer. Nat. Biotechnol. 29, 24-26 (2011).

63. Schindelin, J. et al. Fiji: an open-source platform for biological-image analysis. Nat. Methods 9, 676-682 (2012).

64. Lee, D. W., Hong, C. P. \& Kang, H. A. An effective and rapid method for RNA preparation from non-conventional yeast species. Anal. Biochem. 586, 113408 (2019).

65. Aranda, P. S., LaJoie, D. M. \& Jorcyk, C. L. Bleach gel: a simple agarose gel for analyzing RNA quality. Electrophoresis 33, 366-369 (2012).

66. Fuchs, B. B., O’Brien, E., Khoury, J. B. E. \& Mylonakis, E. Methods for using Galleria mellonella as a model host to study fungal pathogenesis. Virulence 1, 475-482 (2010).

\section{Acknowledgements}

We thank J. Andrew Alspaugh (Duke University) for generously donating A. tumefaciens EHA105 and pPZP-NEO. We also express our appreciation to the CDC for making C. auris isolates used in this study available through the Antibiotic Resistance Bank program. NIH T32AI007528 (NIAID), D.J.S.; NIH KAI137299 (NIAID), T.R.O.

\section{Author contributions}

D.J.S. and T.R.O. designed and performed experiments. D.J.S. designed and built all genetic constructs and mutants. D.J.S. performed data analysis and wrote the manuscript. T.R.O. provided project supervision and critical input on manuscript preparation.

\section{Competing interests}

The authors declare no competing interests.

\section{Additional information}

Supplementary information The online version contains supplementary material available at https://doi.org/10.1038/s41467-021-27545-5.

Correspondence and requests for materials should be addressed to Teresa R. O'Meara.

Peer review information Nature Communications thanks the anonymous reviewers for their contribution to the peer review of this work. Peer reviewer reports are available.

Reprints and permission information is available at http://www.nature.com/reprints

Publisher's note Springer Nature remains neutral with regard to jurisdictional claims in published maps and institutional affiliations.

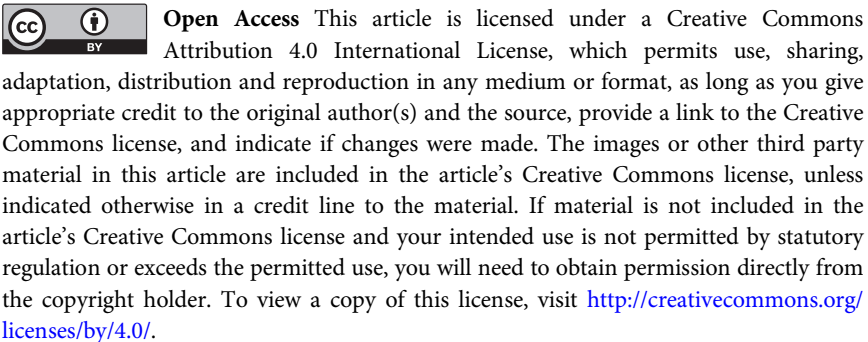
licenses/by/4.0/.

(C) The Author(s) 2021, corrected publication 2022 